\title{
Review of: "A Brazilian Portuguese translation, cultural adaptation and validation of the Arrhythmia- Specific questionnaire in Tachycardia and Arrhythmia (ASTA) health-related quality of life (HRQOL) scale"
}

\section{Raquel Coelho}

Potential competing interests: The author(s) declared that no potential competing interests exist.

The article seeks to fill a gap in the treatment focused on the patient's quality of life through instrument translation, cultural adaptation and validation. The metodology used is in line with the literature and the main necessary steps were fulfilled. The study brings a new scientifically validated instrument that will be useful to guide health care professionals in a patient-centered approach. 